\title{
Incidencia de la Estrategia ECA Y Las Tic en el Desarrollo de Destrezas del Pensamiento en Estudiantes de Secundaria
}

\author{
Impact of ECA Strategy And Ict in the Development of Thinking Skills In High \\ School Students
}

\author{
Gloria Marina Orozco Ferreira \\ Magister en Educación de la Universidad del Norte.E-mail: gloriadeamin@hotmail.com
}

\section{Sandra Villarreal Villa}

Doctorante en Educación de la Universidad del Norte. Decana Facultad de Humanidades, Universidad de la Costa CUC. Magister en Educación, Especialista en Docencia Universitaria, Especialista en Telemática e Informática. Licenciada en Educación.E-mail: svillarreal@cuc.edu.co

\section{Jan Jairo Consuegra Ramírez}

Licenciado en Biología y Química con Maestría en Educación de la Universidad del Norte. E-mail: janconsuegra@hotmail.com

Para citar este artículo: Orozco, G., Acosta, Villareal, S.(2016). Incidencia de la Estrategia ECA Y Las Tic en el Desarrollo de Destrezas del Pensamiento en Estudiantes de Secundaria. Escenarios, 14 (1), p,p.102-116

DOI: http: / / dx.doi.org/10.15665/esc.v14i1.882

Recibido: Marzo 18 de 2016

Aprobado: Abril 19 de 2016

\begin{abstract}
Existe una gran preocupación en la sociedad entorno al desarrollo óptimo de las destrezas de pensamiento, lo cual le impide a los estudiantes cruzar las etapas de sus niveles básicos para poder alcanzar un modelamiento de pensamiento complejo que les permita transferir experiencias a situaciones resolutivas de la vida real, a partir de la toma de decisiones que coadyuven a inferir su aprendizaje significativo por medio de la observación. Esto se ha manifestado en países como Colombia, en donde los estudiantes presentan incoherencias entre su nivel de pensamiento y sus edades. El objetivo de esta investigación fue analizar los efectos de la estrategia de exploración, conceptualización y aplicación (ECA), en ambientes virtuales de aprendizaje. El marco metodológico fue abordado bajo el paradigma cuasi-experimental. Los resultados demostraron que si bien la estrategia (ECA) permite elevar las destrezas de pensamiento en los estudiantes, cuando se usan e incorporan Tecnologías de Información y Comunicación (TIC) y se utilizan sus escenarios virtuales, se potencializa el nivel de logro de esas habilidades, lo cual fue demostrado por las diferencias significativas entre los grupos observados.
\end{abstract}

Palabras Clave: Estrategia de aprendizaje, TIC, pensamiento, destrezas, 


\section{RESUMEN}

There is great concern in society around the optimal development of thinking skills, which prevents students across the stages of their baselines to achieve modeling of complex thinking that allows them to transfer experiences resolute life situations real, from making decisions that help to infer its significant learning through observation. This has manifested itself in countries like Colombia, where students present inconsistencies between their level of thinking and their ages. The objective of this research was to analyze the effects of the exploration strategy, conceptualization and implementation (ECA), in virtual learning environments. The methodological framework was addressed under quasi-experimental paradigm. The results showed that while the ECA strategy can raise thinking skills in students, when used and incorporate Information and Communication Technologies (ICT) and its virtual scenarios are used, the level of achievement of those skills is potentiated, what which it was demonstrated by significant differences between groups observed

Keywords: Learning strategy, ICT, thought, skills.

\section{INTRODUCCIÓN}

De los dilemas más significativos en el ámbito educativo se destaca la preocupación del bajo nivel que muchos alumnos demuestran sobre sus destrezas de pensamiento, debido a la incongruencia entre sus competencias en comparación con sus edades, mermando su capacidad resolutiva para su vida contextual. De esta forma, emergen aportes de proponentes de la corriente del pensamiento crítico; por una parte, Sánchez (2001) quien en su teoría concibe las destrezas básicas de pensamiento como procesos cuya operación final es la síntesis como manifestación del acto mental, Priestley (1996) en cambio destaca la percepción como habilidad inicial del pensamiento y la elaboración de un resumen como habilidad final del mismo; Villarini (1991), concibe la observación como destrezas de pensamiento inicial hasta la solución de problemas en término de aprendizaje significativo, entendido como producto final del proceso de pensamiento. Ellos se han preocupado en buscar soluciones factibles a la problemática suscitada.

La propuesta de Villarini (2003) reconocida como ECA, se ha diseminado por Latinoamérica; por tanto, ha podido contextualizarse como una estrategia que permite organizar el proceso de aprendizaje en fases que dan pertinencia y activan el potencial de crecimiento intelectual del estudiante.

La situación problema de los estudiantes que aún no alcanzan maximizar y potencializar su niveles de destreza de pensamiento, sigue latente en el contexto latinoamericano, como lo refieren (Iriarte, Cantillo y Polo, 2000). Es por ello que el objetivo de esta investigación fue analizar los efectos de la estrategia de exploración, conceptualización y aplicación (ECA), en ambientes virtuales de aprendizaje.

Con estudiantes de un colegio de educación media académica en la ciudad de Barranquilla, escogidos mediante una muestra intencional no probabilística. El trabajo se desarrolló en etapas, que permitieron reconocer, a través de sus resultados, que la estrategia ECA permite elevar las destrezas de pensamiento en los estudiantes, pero aunadas a ambientes virtuales de aprendizaje, se potencializa el nivel de logro de esas habilidades, lo cual fue demostrado por las diferencias significativas entre los grupos observados.

Las habilidades y niveles de pensamiento en la educación

Existe una gran preocupación relacionada al desarrollo de las habilidades de pensamiento, especialmente cuando se compara con las propuestas evolutivas, como la de Piaget y Vygotsky, donde los niveles de pensamiento de los estudiantes en primaria, secundaria y hasta pregrado no están acordes con sus edades; es más, están muy por debajo de los niveles esperados. Jóvenes de la secundaria (Iriarte \& Cols., 1986), universitarios 
(Iriarte \& Cantillo, 1986) Colombianos, y en sí latinoamericanos (Ramírez \& Ramos, 1997), no alcanzan el pensamiento formal en el intervalo de tiempo propuesto para ello.

Esta problemática, vista desde un panorama más amplio, sigue generado inquietudes, investigaciones y propuestas a nivel mundial. Dentro de estas propuestas se distingue la estrategia de enseñanza ECA (exploración, conceptualización y aplicación), que permite organizar el proceso de aprendizaje en fases que dan pertinencia y activan el potencial de crecimiento intelectual del estudiante, precisa objetivos instrucciones a la luz de los intereses y necesidades de los estudiantes y además, organiza el proceso de enseñanza simultáneamente con un proceso de evaluación y retro comunicación (Villarini, 2003).

Desde la Psicología Genética, se exalta a Jean Piaget y Lev Vygotsky (citados por Palmer, 2000), quienes analizaron la forma como se construye el conocimiento en el niño. Para Piaget, es el producto entre asimilación y acomodación; y de los diversos estadios del pensamiento, formas operatorias o simbólicas, para Vygotsky, el conocimiento es contenido y actividad en las que el sujeto emplea instrumentos como son la asimilación y la acomodación. El sujeto emplea instrumentos como la asimilación y la acomodación, señalados por Piaget y medios de la naturaleza sociocultural y del lenguaje señalados por Vygotsky que juegan un papel fundamental en el proceso.

Sánchez (1992), concibe las destrezas básicas de pensamiento como procesos cuya operación final es la síntesis como manifestación del acto mental, que requiere agotar en forma consciente otros procesos como la observación y descripción, identificación de características, establecimiento de diferencias y semejanzas, comparación y relación, clasificación, análisis y síntesis.

De Bono (1995), propone asumir el pensamiento como una destreza que posee el ser humano para utilizar el potencial de su inteligencia. Para él, quien piensa bien no se queda en el plano pasivo de ver y juzgar sino de ver- explotar y juzgar. Cuando falta el "explorar" el pensamiento es defectuoso citado por (Romero, Rodríguez y Ramírez, 2003). Igualmente, Novak \& Gowin
(1993), expresan que una de las condiciones para que se dé el aprendizaje significativo es que el aprendiz presente una predisposición para aprender, es decir una experiencia afectiva y que el material de aprendizaje sea potencialmente significativo, lo que se constituye el fundamento sobre el cual se argumenta la integración constructiva del pensamiento. Para que se apliquen los procesos de pensamiento, se requiere de una motivación (sentimiento, emociones, intereses y valores), de estructuras conceptuales (conceptos) y de una serie de métodos o procedimientos (destrezas y habilidades), todo ello inmerso en un contexto histórico-social- cultural y acompañado de la autorreflexión, dándole significado para ser transferido.

Para Villarini (1991) el pensamiento es la capacidad para procesar información y construir conocimiento, combinando representaciones, operaciones y actitudes mentales en forma automática, sistemática, creativa o crítica para producir creencias, conocimientos, plantear problemas y encontrar soluciones. Igualmente, considera que para comprender las diferentes destrezas de pensamiento, se debe partir de tres (3) niveles de funcionamiento mental consciente del proceso de pensamiento: a) automático, se hacen las cosas sin pensar; b) sistemático, se detiene a pensar y; c) pensamiento crítico, se examina la actividad y el proceso de pensamiento, analizando y evaluando las operaciones, conceptos y actitudes en su relación con la realidad que se vive. Se establece una secuencia y niveles en las destrezas de pensamiento que se refiere al orden en que ocurre la producción de nuevos conocimientos a través del pensamiento denominadas destrezas básicas o simples y destrezas complejas (Villarini, 1991).

En la propuesta de Villarini, se evidencia como éste complementa la teoría de Piaget con la de Vygotsky, en donde no solo la genética y la evolución de los factores determinan el desarrollo del pensamiento, sino que el contexto socio-cultural del individuo interviene en dicho proceso (Rodriguez, 1995).

Las destrezas básicas o simples, se limitan a un procesamiento superficial de los estímulos o información. A este nivel la información es me- 
ramente comprendida en su forma literal o inmediata. Sin establecer otras relaciones pero son necesarias para que se puedan desarrollar las destrezas complejas.

Complementariamente, cabe resaltar que las destrezas avanzadas o Complejas, procesan la información a un nivel mayor de profundidad o complejidad (relaciones y conexiones) como inferir, analizar, solucionar problemas y tomar decisiones. Ellas requieren de la combinación de varias destrezas y del empleo de una multiplicidad de conceptos, tal como se evidencia en los aportes de Batista, Salazar y Febres Cordero (2001).

En el modelo de pensamiento sistemático, se reconocen doce (12) operaciones o destrezas generales. Cada destreza es un procedimiento para el manejo de la información. El fomento del desarrollo del pensamiento implica conocer el procedimiento que caracteriza a cada destreza y ayudar al estudiante a desarrollar lo en forma efectiva. Todo este proceso se da según la complejidad de la información que se va a abarcar.

Por consiguiente, se requiere de estudiantes conscientes de las destrezas de pensamiento que poseen y de la necesidad de entrenarlas para ser cada día individuos más competentes tanto en su desempeño académico, como en lo laboral y en lo social en un contexto global donde pueda intercambiar y fortalecer información que enriquezca significativamente los nuevos saberes visibilizándose en la forma como da respuesta a las necesidades actuales y de los resultados de las diferentes pruebas aplicadas a nivel nacional SABER e internacional Informe del Programa Internacional para la Evaluación de Estudiantes (PISA) y Estudio de las Tendencias en Matemáticas y Ciencias (TIMSS), que miden estas competencias.

La Estrategia ECA y su articulación funcional con los ambientes virtuales de aprendizaje

La estrategia ECA garantiza el desarrollo de las destrezas de pensamiento en los estudiantes mediante la implementación de tres fases: Exploración (E), Conceptualización (C) y Aplicación (A) en las que se definen actividades programadas y responsabilidades de profesores y estudiantes en el proceso de enseñanza-aprendizaje.
En ese sentido, a través de las actividades de la fase de exploración se activa en el estudiante el conocimiento, destrezas y actitudes previas que tiene y necesita para poder procesar o asimilar la nueva información que el maestro le presentará o ayudará a conseguir a través de las actividades programadas. Estas actividades plantean situaciones o problemas que provocan la discusión, le permiten al profesor realizar un diagnóstico acerca de los conocimientos, actitudes y destrezas con los que cuenta el estudiante y se logra que el estudiante relacione lo que va a aprender con sus experiencias y aprendizajes previos. El maestro juega un papel de facilitador haciendo que los estudiantes progresen de la observación, a la comparación, hasta las destrezas complejas.

En la fase de conceptualización, se estimula al estudiante a desarrollar o enriquecer el concepto o destreza activado en la fase de exploración, descubriendo o analizando para qué sirve el concepto, la estructura del concepto brindando ejemplos positivos y negativos del mismo mediante la guía docente.

Bajo esta estrategia, el proceso de enseñanza puede tener lugar a través de dos métodos: el expositivo y el interactivo. En el primero, el maestro define el concepto y a partir del ejemplo, explica elementos, criterios, importancia y utilidad mientras que en el segundo se parte del ejemplo hasta alcanzar la definición del concepto. En ambos métodos se clarifican dudas y se asignan tareas para aplicar el concepto mediante una práctica guiada señalando características similares que permite la construcción de una definición.

En la fase de aplicación, se consolida el desarrollo del concepto, la información presentada por el maestro(a) ha sido transformada en una estructura mental de la cual el estudiante se sirve para procesar información. Si en efecto el estudiante ha desarrollado el concepto, éste estará en capacidad de aplicar o transferir lo aprendido a nuevas situaciones y problemas.

El maestro(a) tiene que asegurarse que el estudiante sea consciente del proceso de pensamiento que llevó a cabo en el desarrollo del ejercicio, reconociendo sus debilidades, fortalezas y limitaciones que se presentaron y que le sirven como 
fundamento para un nuevo proceso de aprendizaje.

Para la implementación de esta estrategia, la investigación se centra en el desarrollo de las destrezas de pensamiento en ambientes virtuales mediados por las Tecnologías de la Información y la Comunicación - TIC. Desde esta perspectiva, las TIC constituyen una herramienta muy poderosa e importante para que la educación cree y recree ambientes que permitan formar seres humanos conscientes de su realidad dentro del contexto mundial o globalizado, comprometidos con sus deberes ciudadanos y responsabilidades civiles, miembros de un entorno cambiante y con las habilidades y destrezas para adaptarse, apropiarse y participar en estos ambientes en todo momento de manera positiva (Gisbert, 2000).

La importancia del uso de las TIC en el contexto contemporáneo se ha identificado en una amplia gama de estudios y es así como el Colectivo Educación Infantil y TIC (2014) resalta la necesidad permanente de innovar en el diseño y desarrollo de recursos educativos digitales para la educación, que sean ajustados a las necesidades, nivel y entorno educativos.

Por otra parte, uno de los problemas que presenta la implementación de las TIC, es que a pesar de su gran potencial de enriquecer los ambientes de aprendizaje. No desarrollan por sí mismas el pensamiento crítico y que esta responsabilidad le corresponde al docente, debido a la forma en que estos decidan usarlas, lo cual determinará si las TIC ayudan o inhiben el desarrollo de habilidades para el pensamiento crítico en ambientes virtuales de aprendizaje. Por lo tanto los docentes deben asegurarse de hacer una planeación adecuada para que sus estudiantes utilicen todo el potencial que tienen estas herramientas, teniendo en cuenta que el éxito o fracaso de las innovaciones depende, en gran parte, de la forma como los diferentes actores educativos interpretan, redefinen, filtran y dan forma a los cambios propuestos. Las innovaciones en educación tienen ante sí como principal reto los procesos de adopción por parte de las personas, los grupos y las instituciones. (Salinas, 2004)

En acuerdo con Crompton y Thompson (2010) los maestros que empoderan a los estudiantes para asumir una participación activa en un wiki, blog u otras herramientas de la Web 2.0 están en el camino correcto, estas herramientas estimulan permanentemente el pensamiento crítico. De hecho, algunas de estas herramientas exigen ese nivel de reflexión, puesto que editar, revisar, criticar y comentar, hace parte integral de su uso. Como los estudiantes no tienen que esperar a regresar al salón de clase para usar sus habilidades de pensamiento crítico, se involucran profundamente en el aprendizaje y eventualmente se convierten en aprendices de por vida, que evalúan información como consumidores activos de conocimiento.

Es así como se identifican diferentes aplicaciones que sugieren la articulación entre las destrezas cognitivas y el uso de los escenarios virtuales de aprendizaje en los trabajos de Albano (2012) quien profundiza sobre los conocimientos, destrezas y competencias a partir de un modelo diseñado para aprender el mundo de las matemáticas en un entorno virtual; en conjunción con los aportes de Baptista (2010) relacionados con los modelos de aprendizaje de una segunda lengua.

Las herramientas del conocimiento, como se le llaman a las TIC en la Educación, permiten la construcción de éste en el estudiante, más no la reproducción de los conocimientos de los docentes. Estas permiten una educación constructivista ya que se ocupan del proceso de cómo construimos el conocimiento. Cuando los estudiantes elaboran estrategias mediatizadas por las TIC están construyendo su propia conceptualización de la organización de un dominio de contenidos.

\section{Método}

En congruencia con el propósito de analizar los efectos diferenciales de la estrategia ECA en los niveles en las destrezas del pensamiento de los estudiantes con y sin el uso del escenario de los ambientes virtuales de aprendizaje, a continuación se describen las especificaciones metodológicas contempladas para el desarrollo del presente estudio.

\section{Diseño}

Se abordó desde el paradigma positivista en el marco de una investigación cuantitativa, en la cual se empleó un diseño cuasi-experimental con dos grupos (experimental y control) e incorporó 
mediciones de tipo (pretest - postest). El enfoque de la investigación cuantitativa, busca determinar, mediante la recolección objetiva de datos, si existe diferencia significativa en los efectos de la implementación de ECA en ambientes virtuales de aprendizaje apoyada en las TIC en el desarrollo de las destrezas de pensamiento y categorías de conocimiento en un grupo de estudiantes de Educación Media Académica. Éste abordaje coincide con los planteamientos de Hernández, Fernández y Baptista (2006), en el sentido de que una investigación cuantitativa se caracteriza por "usar la recolección de datos para probar hipótesis, con base en la medición numérica y el análisis estadístico, para establecer patrones de comportamiento y probar teorías" (p.5).

\section{Participantes}

La muestra fue conformada gracias a la participación de 41 estudiantes de $10^{\circ}$ grado, con edades comprendidas entre los 14 y 17 años, estrato socioeconómico medio- alto de un colegio de la ciudad de Barranquilla; quienes fueron escogidos bajo el escenario de grupos intactos o previamente asignados. Cabe resaltar que los participantes a partir de las estrategias curriculares de la institución educativa ya contaban con entrenamiento previo en competencias básicas en el uso de TIC.

Ambos grupos intactos de $10^{\circ}$ grado fueron seleccionados para la investigación en el contexto académico de la asignatura de química inorgánica, el primer grupo (GE1: ECA) contaba con 22 estudiantes y el segundo grupo (GE2: ECA + TIC) estaba conformado por 19 estudiantes. Fue valiosa la disponibilidad de los profesores, quienes también participaron en dos talleres de formación uno relacionado con el entrenamiento en el uso de la estrategia ECA y el otro, en uso de TIC con un número total en formación de 120 horas cada uno; además del consentimiento voluntario de las autoridades escolares y de la disposición de todos los recursos.

El diseño planteado es cuasi-experimental de medición pretest y después, con dos grupos experimentales: uno intervenido con ECA y otro intervenido con ECA apoyada en TIC. Se realizó un diagnóstico previo para determinar las destrezas de pensamiento que manejaban los jóvenes y el nivel de conocimiento en la asignatura determinada (química inorgánica). Una vez obtenida la información, se seleccionó la muestra para ambos grupos experimentales, según la conveniencia de los investigadores. De tal manera que las relaciones quedaron establecidas, tal como se especifica a continuación en la tabla 1.

Técnicas e Instrumentos La técnica utilizada fue una encuesta personal, para la recolección de la información, en donde "Existe un contacto directo entre el entrevistador y el entrevistado" (Hernández, Fernández, \& Baptista, 2006), el instrumentos para la evaluación de las variables escogidas fueron implementados dos cuestionarios de preguntas abiertas validados por juicio de expertos. El primero correspondiente a determinar el nivel de destreza de pensamiento en el que se encontraban los estudiantes pretest y postest de ser intervenidos con ECA y ECA apoyada en TIC, y el segundo cuestionario corresponde a una prueba objetiva de los niveles de conocimiento en Química, específicamente en el tema de Nomenclatura, pretest y postest de ser intervenidos ambos grupos observados. La prueba de nivel de destreza de pensamiento fue diseñada según actividades propuesta por Sánchez (2003) y la prueba objetiva de conocimiento según los dos primeros niveles de la taxonomía de Bloom en el contenido de química inorgánica en particular Nomenclatura química.

Procedimiento Inicialmente se formularon las especificaciones del proceso de intervención con

Tabla 1. Especificaciones muestrales del diseño cuasi-experimental

\begin{tabular}{|c|c|c|c|c|}
\hline Grupo & Asignación & $\begin{array}{c}\text { Medición } \\
\text { antes }\end{array}$ & Aplicación & $\begin{array}{c}\text { Medición } \\
\text { después }\end{array}$ \\
\hline GE1: Grupo ECA & Intencional & $\mathrm{O} 1$ & $\mathrm{X} 1$ & $\mathrm{O} 2$ \\
\hline GE2: Grupo ECA + TIC & Intencional & $\mathrm{O} 3$ & $\mathrm{X} 2$ & $\mathrm{O} 4$ \\
\hline
\end{tabular}

Fuente: Elaboración propia 
la estrategia ECA incorporada en el ambiente virtual de aprendizaje. El diseño cuasi-experimental fue argumentado inicialmente con el análisis del constructo teórico-científico y adicionalmente se fortaleció metodológicamente con las fases previas de diseño y validación de los instrumentos de recolección de datos, entrenamiento de docentes e investigadores, solicitud de consentimiento informado para la selección de los participantes y asignación a las dos condiciones (experimental y control), diagnóstico previo (pretest), intervención con la estrategia ECA bajo el ambiente virtual de aprendizaje, diagnóstico final (postest) y análisis de resultados.

Dado los hallazgos, luego de aplicar el cuestionario diagnóstico para determinar el nivel de destreza de pensamiento y nivel de conocimiento en los estudiantes de la muestra y garantizar la homogeneidad de ésta al ser seleccionada, fue necesario redefinir las destrezas de pensamiento y niveles de conocimiento en observar - comparar y en recordar - comprender al no alcanzar los estudiantes los niveles esperados. La Estrategia ECA fue aplicada en el GE1 y la Estrategia ECA apoyada con TIC en el GE2. Cabe destacar que por cada tema a desarrollar era indispensable asegurarse que la clase se desarrollara según las fases de exploración, conceptualización y aplicación, que plantea la estrategia.

Sesión 1. Fase de exploración: Activar la destreza y el concepto, mediante situación pertinente provocadora. - Facilitar el uso de la destreza. Observar el uso de la destreza en el estudiante.n - Evaluar y diagnosticar el uso de la destreza en los estudiantes.

Sesión 2. Fase de conceptualización - Guiar al estudiante en el uso de la destreza, para la ejecución de la tarea. - Observar la ejecución de la destreza en el estudiante. - Retroalimentar al estudiante con respecto a la ejecución de la destreza.
Sesión 3. Fase de aplicación: - Asignar una nueva tarea. - Observar la ejecución de la destreza en el estudiante. - Evaluar y diagnosticar la ejecución de la destreza.

En el grupo experimental con ECA apoyado en TIC, los procedimientos fueron aplicados directamente a los estudiantes usando tecnologías tales como herramientas de visualización, de modelado dinámico y de comunicación y colaboración en el curso de Química Interactiva diseñado en la plataforma Moodle.

\section{Resultados}

Para analizar los datos obtenidos se aplicó una prueba paramétrica, $\mathrm{T}$ de Student de diferencia de medias para muestras relacionadas, con previo cumplimiento de los supuestos de normalidad que esta prueba requiere. La interpretación de los resultados permitió considerar en conjunto y en forma particular las destrezas de pensamiento (Observar- Comparar) y las categorías de conocimiento (Recordar-Comprender) en un grupo de estudiantes intervenido con ECA (GE1) y otro grupo intervenido con ECA apoyada en TIC (GE2). Los datos fueron organizados atendiendo los criterios expresados en la Tabla 2.

Cada rango detalla el nivel de desempeño (Muy bajo, Bajo, Medio, Alto, Muy Alto) alcanzado por los estudiantes que respondieron de manera correcta a las preguntas de la evaluación dependiendo del tipo de destreza de pensamiento y la categoría de conocimiento adquiridos.

En la Tabla 3, se aprecian los estadísticos descriptivos derivados de las medidas relacionadas para los grupos (GE1 y GE2) intervenidos con la estrategia ECA y la segunda condición con la estrategia ECA apoyada en TIC. Se observa que las medias obtenidas luego de la intervención tienden a ser superiores frente a las medias obtenidas pretest de la misma.

Tabla 2. Criterios de valoración del desempeño

\begin{tabular}{|c|c|c|c|c|}
\hline Muy Bajo & Bajo & Medio & Alto & Muy Alto \\
\hline 0 a $20 \%$ & 21 a $40 \%$ & 41 a $60 \%$ & 61 a $80 \%$ & 81 a $100 \%$ \\
\hline
\end{tabular}

Fuente: Elaboración propia 
Tabla 3. Estadísticos Descriptivos Comparaciones Relacionadas (pretest - postest)

\begin{tabular}{|c|c|c|c|c|c|}
\hline \multicolumn{6}{|c|}{ ESTADISTICOS DE MUESTRAS RELACIONADAS } \\
\hline GRUPO & & & MEDIA & DESV. TIPICA & ERROR TIP DE LA MEDIA \\
\hline \multirow{8}{*}{ GE1: ECA } & PAR 1 & Pensamiento Observar pretest ECA & 53,30 & 27,67 & 5,89 \\
\hline & & Pensamiento Observar postest ECA & 68,18 & 14,23 & 3,03 \\
\hline & $\overline{\text { PAR } 2}$ & Pensamiento Comparar pretest ECA & 36,89 & 13,19 & 2,81 \\
\hline & & Pensamiento Comparar postest ECA & 57,27 & 9,42 & 2,00 \\
\hline & PAR 3 & Conocimiento Recordar pretest ECA & 53,63 & 21,10 & 4,49 \\
\hline & & Conocimiento Recordar postest ECA & 81,61 & 9,76 & 2,08 \\
\hline & PAR 4 & Conocimiento Comprender pretest ECA & 38,42 & 25,70 & 5,47 \\
\hline & & Conocimiento Comprender postest ECA & 73,96 & 27,38 & 5,83 \\
\hline \multirow{8}{*}{$\begin{array}{c}\text { GE2: } \\
\text { ECA + TIC }\end{array}$} & PAR 1 & Pensamiento Observar pretest ECA + TIC & 46,88 & 26,97 & 6,18 \\
\hline & & Pensamiento Observar postest ECA + TIC & 93,77 & 12,51 & 2,87 \\
\hline & PAR 2 & Pensamiento Comparar pretest ECA + TIC & 42,63 & 10,51 & 2,41 \\
\hline & & Pensamiento Comparar postest ECA + TIC & 62,98 & 6,90 & 1,58 \\
\hline & PAR 3 & Conocimiento Recordar pretest ECA + TIC & 69,13 & 20,91 & 4,79 \\
\hline & & Conocimiento Recordar postest ECA + TIC & 85,88 & 10,48 & 2,40 \\
\hline & PAR 4 & Conocimiento Comprender pretest ECA + TIC & 47,84 & 25,68 & 5,89 \\
\hline & & Conocimiento Comprender postest ECA + TIC & 89,47 & 14,92 & 3,42 \\
\hline
\end{tabular}

Fuente: Elaboración propia

Tabla 4. Análisis correlacional de las muestras relacionadas (pretest - postest)

\begin{tabular}{|c|c|c|c|c|}
\hline GRUPO & & & CORRELACION & SIG \\
\hline \multirow{4}{*}{ GE1: ECA } & PAR 1 & $\begin{array}{l}\text { Pensamiento - Observar } \\
\text { pretest ECA/ postest ECA }\end{array}$ & 0,49 & $0,02 *$ \\
\hline & PAR 2 & $\begin{array}{l}\text { Pensamiento- Comparar } \\
\text { pretest ECA/ postest ECA }\end{array}$ & 0,54 & $0,01 *$ \\
\hline & PAR 3 & $\begin{array}{l}\text { Conocimiento- Recordar } \\
\text { pretest ECA/ postest ECA }\end{array}$ & 0,36 & 0,10 \\
\hline & PAR 4 & $\begin{array}{l}\text { Conocimiento-Comprender } \\
\text { pretest ECA/ postest ECA }\end{array}$ & 0,09 & 0,71 \\
\hline \multirow{4}{*}{ GE2: ECA + TIC } & PAR 1 & $\begin{array}{l}\text { Pensamiento- Observar } \\
\text { pretest ECA + TIC/ postest ECA + TIC }\end{array}$ & 0,03 & 0,91 \\
\hline & PAR 2 & $\begin{array}{l}\text { Pensamiento- Comparar } \\
\text { pretest ECA + TIC/ postest ECA+TIC }\end{array}$ & 0,14 & 0,56 \\
\hline & PAR 3 & $\begin{array}{l}\text { Conocimiento- Recordar } \\
\text { pretest ECA + TIC/ postest ECA+TIC }\end{array}$ & 0,60 & 0,80 \\
\hline & PAR 4 & $\begin{array}{l}\text { Conocimiento Comprender } \\
\text { pretest ECA + TIC /después ECA+TIC }\end{array}$ & 0,43 & 0,67 \\
\hline
\end{tabular}

Nota: Las correlaciones estadísticamente significativas al 95\% se encuentran identificadas con $\left(^{*}\right)$.

Fuente: Elaboración propia

En la Tabla 4, se observan las correlaciones de muestras que indica que los estudiantes obtuvieron puntajes altos en las destrezas de pensamiento y categorías de conocimiento luego de ser intervenidos atendiendo al nivel de significancia, es decir menores al nivel de confianza establecido previamente de 0,05.

En las destrezas de pensamiento, es de anotar que los resultados muestran que no existen diferencias significativas pretest y postest de ser intervenidos con la estrategia ECA (POBAE $=53,30$; $\mathrm{POBDE}=68,18 ; \mathrm{p}>0.05$ ). Por consiguiente, en los estudiantes no se evidencia cambio en su capaci- dad para fijar la atención en los atributos o características de los objetos. Caso contrario, en el grupo intervenido con ECA apoyado con TIC cuyos resultados permiten afirmar la alta capacidad de observación en los estudiantes (POBAET $=46,88$; POBDET $=93,77 ; \mathrm{p}<0.05)$. Atendiendo las demás destrezas de pensamiento y categorías de conocimientos consideradas en esta investigación en todas y cada una de ellas tanto en el grupo GE1 y GE2 se evidencia diferencias significativas:

Destrezas de pensamiento: "Comparar" Grupo GE1 (PCOMAE=36,89; PCOMDE $=57,27$; $\mathrm{p}<0,05) ; \quad$ GE2 $\quad($ PCOMAET $=42,63 ; \quad$ PCOM- 
$\mathrm{DET}=62,98 ; \mathrm{p}<0,05)$.Esto nos indica que los estudiantes mejoraron en su capacidad para comparar contrastando dos o más datos, estableciendo semejanzas y diferencias y relaciones de equivalencia entre datos o entre situaciones dadas.

En las categorías de conocimiento comprender y recordar los resultados muestran la existencia de diferencias significativas; por lo anterior la aplicación de la estrategia tiene una gran influencia en los estudiantes al mejorar la forma de evocar material aprendido como hechos, términos, conceptos básicos y respuestas; así mismo la capacidad para entender hechos e ideas, organizando, comparando, interpretando y haciendo descripciones y exposiciones de ideas principales.

En la tabla 5 se muestran los datos que informan sobre qué tan significativo fueron los cambios logrados en desarrollo de pensamiento y en categorías de conocimiento de los estudiantes. De acuerdo con la tabla, se produjeron diferencias altamente significativas en los grupos intervenidos con ECA pero fue mayor aún en los grupos intervenidos con ECA apoyados en TIC. Estos resultados indican que fue notoria la mediación de las TIC para alcanzar los niveles de destrezas y categorías de conocimiento propuesta.

En las destrezas de pensamiento, es de anotar que los resultados muestran que no existen diferencias significativas pretest y postest de ser intervenidos con la estrategia $\mathrm{ECA}(\mathrm{POBAE}=53,30$; $\mathrm{POBDE}=68,18 ; \mathrm{p}>0.05)$. Por consiguiente, en los estudiantes no se evidencia cambio en su capacidad para fijar la atención en los atributos o características de los objetos. Caso contrario, en el grupo intervenido con ECA apoyado con TIC cuyos resultados permiten afirmar la alta capacidad de observación en los estudiantes (POBAET $=46,88$; POBDET=93,77; $\mathrm{p}<0.05)$. Atendiendo las demás destrezas de pensamiento y categorías de conocimientos consideradas en esta investigación en todas y cada una de ellas tanto en el grupo GE1 y GE2 se evidencia diferencias significativas:

Destrezas de pensamiento: "Comparar" Grupo GE1 (PCOMAE=36,89; PCOMDE=57,27; $\mathrm{p}<0,05) ; \quad$ GE2 $\quad$ (PCOMAET $=42,63 ; \quad$ PCOM$\mathrm{DET}=62,98 ; \mathrm{p}<0,05)$. Esto nos indica que los estudiantes mejoraron en su capacidad para comparar contrastando dos o más datos, estableciendo semejanzas y diferencias y relaciones de equivalencia entre datos o entre situaciones dadas.

Tabla 5. Diferencias estadísticas: Pruebas T (pretest y postest) para muestras relacionadas

\begin{tabular}{|c|c|c|c|c|c|c|c|c|}
\hline & & & \multicolumn{6}{|c|}{$95 \%$ intervalo } \\
\hline \multirow{2}{*}{\multicolumn{3}{|c|}{ GRUPO }} & & & con & nza & & \\
\hline & & & MEDIA & DESV TIP & INF & SUP & $\mathbf{t}$ & SIG. BILATERAL \\
\hline \multirow[t]{4}{*}{ GE1: ECA } & PAR 1 & $\begin{array}{l}\text { Pensamiento Observar } \\
\text { pretest ECA/ postest ECA }\end{array}$ & 14,87 & 36,85 & 1,46 & 31,21 & 1,8 & .072 \\
\hline & PAR 2 & $\begin{array}{l}\text { Pensamiento Comparar } \\
\text { pretest ECA/ postest ECA }\end{array}$ & 20,37 & 19,93 & 11,53 & 29,21 & 4,7 & $.000^{*}$ \\
\hline & PAR 3 & $\begin{array}{l}\text { Conocimiento Recordar } \\
\text { pretest ECA/ postest ECA }\end{array}$ & 27,97 & 19,85 & 19,17 & 36,77 & 6,6 & $.000^{*}$ \\
\hline & PAR 4 & $\begin{array}{l}\text { Conocimiento Comprender } \\
\text { pretest ECA/ postest ECA }\end{array}$ & 35,53 & 35,91 & 19,61 & 51,46 & 4,6 & $.000^{*}$ \\
\hline \multirow[t]{4}{*}{ GE2: ECA+TIC } & PAR 1 & $\begin{array}{l}\text { Pensamiento Observar } \\
\text { pretest ECA + TIC/ postest } \\
\text { ECA + TIC }\end{array}$ & 46,88 & 30,03 & 32,41 & 61,36 & 6,8 & $.000^{*}$ \\
\hline & PAR 2 & $\begin{array}{l}\text { Pensamiento Comparar } \\
\text { pretest ECA + TIC/ postest } \\
\text { ECA+TIC }\end{array}$ & 20,35 & 13,36 & 13,91 & 26,79 & 6,6 & $.000^{*}$ \\
\hline & PAR 3 & $\begin{array}{l}\text { Conocimiento Recordar } \\
\text { pretest ECA + TIC/ postest } \\
\text { ECA+TIC }\end{array}$ & 16,74 & 22,83 & 5,74 & 27,75 & 3,2 & $.005^{*}$ \\
\hline & PAR 4 & $\begin{array}{l}\text { Conocimiento Comprender } \\
\text { pretest ECA + TIC } \\
\text { /después ECA+TIC }\end{array}$ & 41,62 & 23,53 & 30,28 & 52,96 & 7,7 & $.000^{*}$ \\
\hline
\end{tabular}

Nota: casi todas las diferencias fueron estadísticamente significativas al 95\% (*)

Fuente: elaboración propia 
Figura 1. GE1. ECA: Destreza de pensamiento "Observar" (pretest - postest)

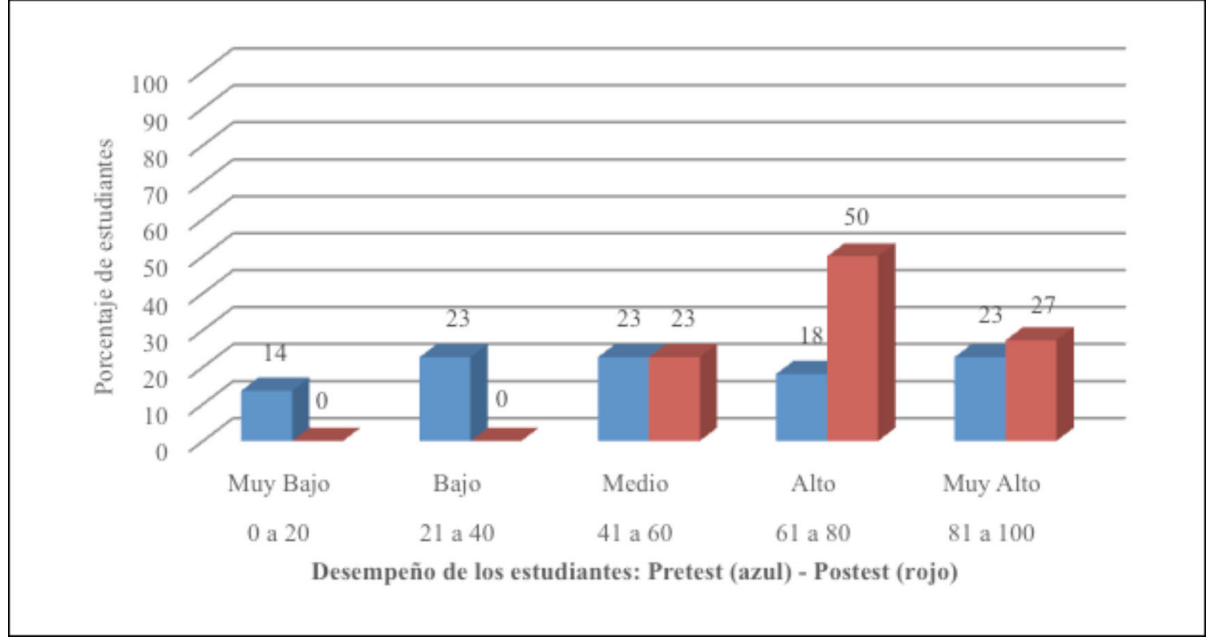

Fuente: Elaboración propia

Figura 2. GE2. ECA+TIC: Destreza de pensamiento "Observar" (pretest - postest)

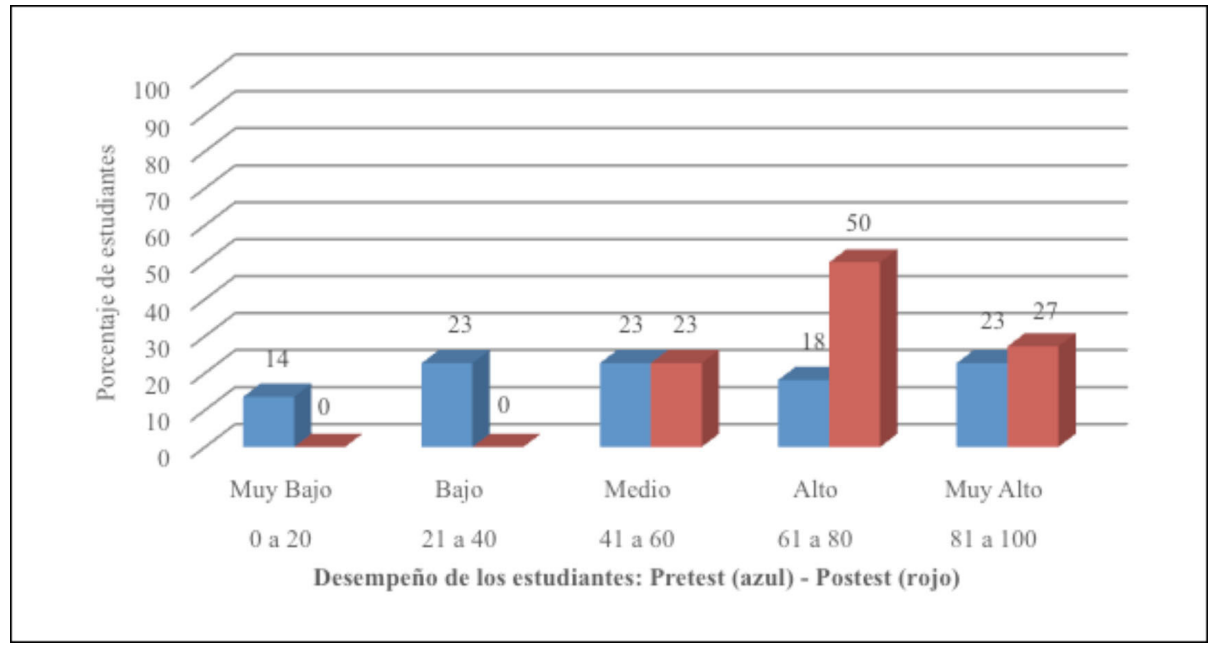

Fuente: Elaboración propia

En las categorías de conocimiento comprender y recordar los resultados muestran la existencia de diferencias significativas; por lo anterior la aplicación de la estrategia tiene una gran influencia en los estudiantes al mejorar la forma de evocar material aprendido como hechos, términos, conceptos básicos y respuestas; así mismo la capacidad para entender hechos e ideas, organizando, comparando, interpretando y haciendo descripciones y exposiciones de ideas principales.

En la tabla 5 se muestran los datos que informan sobre qué tan significativo fueron los cambios logrados en desarrollo de pensamiento y en categorías de conocimiento de los estudiantes. De acuerdo con la tabla, se produjeron diferencias altamente significativas en los grupos intervenidos con ECA pero fue mayor aún en los grupos intervenidos con ECA apoyados en TIC. Estos resultados indican que fue notoria la mediación de las TIC para alcanzar los niveles de destrezas y categorías de conocimiento propuesta.

Las variaciones estimadas en las desviaciones estándar permiten realizar un análisis más detallado del efecto de la intervención en las destrezas 
de pensamiento y categorías de conocimiento de los estudiantes. Se puede notar desviaciones en el postest mayores que en el pretest. Lo anterior permite inferir que hubo un aumento significativo en el grupo de ECA apoyado en TIC en las destrezas de observar y comparar y en las categorías de conocimiento recordar y comprender

En la Figura 1 y Figura 2. Se presenta un análisis de los niveles de destrezas de pensamiento observar y comparar en la medición pretest y postest de aplicada la estrategia ECA comparada con el grupo intervenido por ECA apoyada en TIC.

Una vez intervenidos los grupos con la estrategia ECA y ECA + TIC se determinó que ambas estrategias fueron efectivas en el desarrollo de la destreza de pensamiento Observar. En el grupo intervenido con ECA, los resultados muestran una tendencia mayor en el desempeño de los estudiantes en el nivel "Alto", mientras que el grupo intervenido con ECA + TIC muestra mayor tendencia en el nivel "Muy Alto", estos resultados demuestran que la utilización de la estrategia ECA mediatizada con TIC como elemento de apoyo al evento pedagógico, tiene influencia en los estudiantes, respecto a la destreza de observar, para ubicar y reconocer los grupos funcionales de un función química inorgánica; por lo tanto, existe mayor efectividad en los participantes a quienes se les ha intervenido con ECA + TIC que en aquellos que fueron intervenidos solo con ECA.
En la Figura 3. Se muestra la destreza de pensamiento Comparar, también se determinó la efectividad de ambas estrategias. En el grupo de estudiantes intervenido con ECA la mayor tendencia se presentó en el nivel "Medio" mientras que en el grupo de ECA+TIC, se presentó en el nivel "Alto". Estos resultados muestran que la utilización de la estrategia ECA mediatizada por TIC genera mejor desempeño toda vez que se desee desarrollar la destreza para comparar las características de los objetos o sustancias en términos de sus semejanzas y diferencias, teniendo en cuenta los sistemas de nomenclatura que establece la IUPAC.

En las Figuras 5, 6. Se muestran los análisis de los niveles de categorías de conocimiento recordar y comprender en la medición pretest y postest de aplicada la estrategia ECA comparada con el grupo intervenido por ECA apoyada en TIC

Con respecto a la categoría de conocimiento Recordar se determinó que ambas estrategias fueron efectivas en el desarrollo de la misma. Los niveles de desempeño "Muy Alto" y "Alto" fue la tendencia del grupo intervenido con ECA +TIC y del ECA éste último con un ligero porcentaje (5\%) en nivel "Medio" de desempeño en esta categoría. Lo que significa que la aplicación de ambas estrategias tiene una gran influencia en los estudiantes al mejorar la forma de evocar el material aprendido con anterioridad como características y / o reglas de los sistemas de nomenclaturas establecidos por la IUPAC, para los

Figura 3. GE1. ECA: Destreza de pensamiento “Comparar" (pretest - postest)

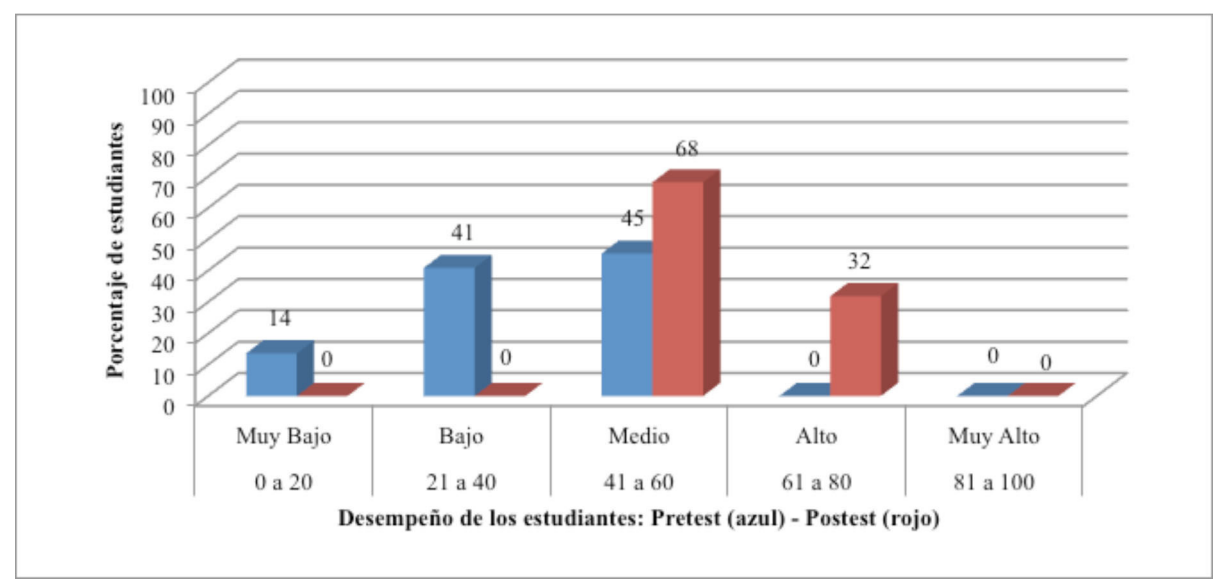

Fuente: Elaboración propia 
Figura 4. GE2. ECA+TIC: Destreza de pensamiento "Comparar" (pretest - postest)

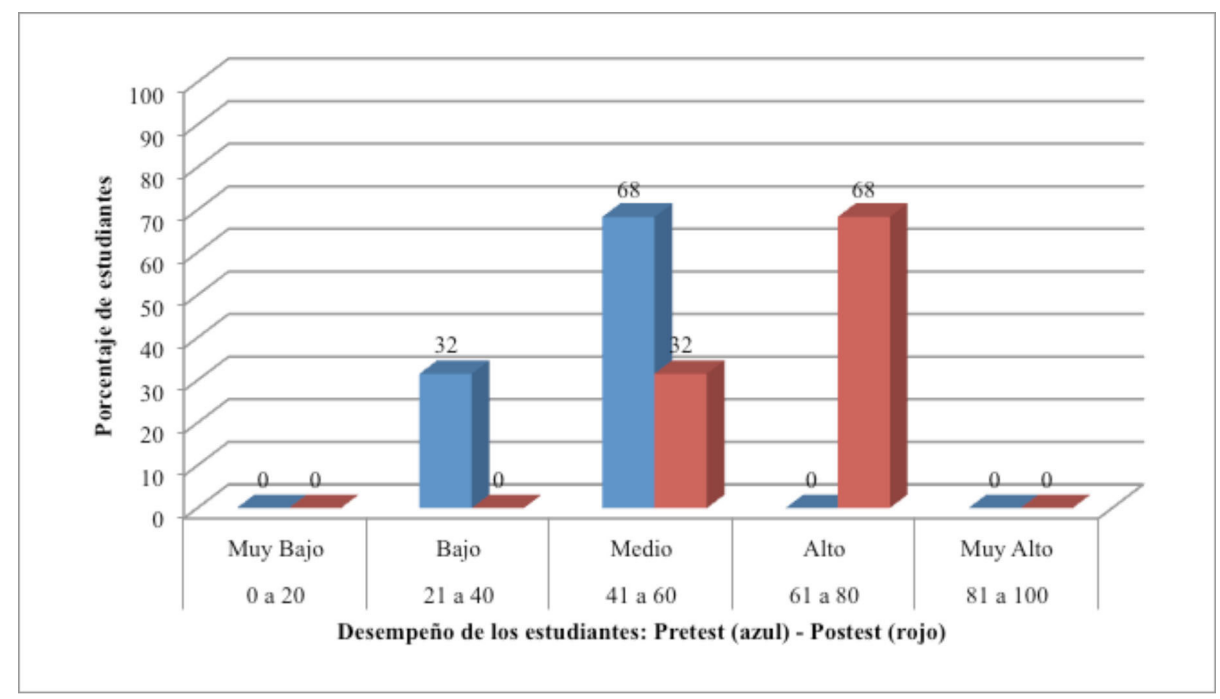

Fuente: Elaboración propia

Figura 5. GE1. ECA: Destreza de pensamiento "Recordar" (pretest - postest)

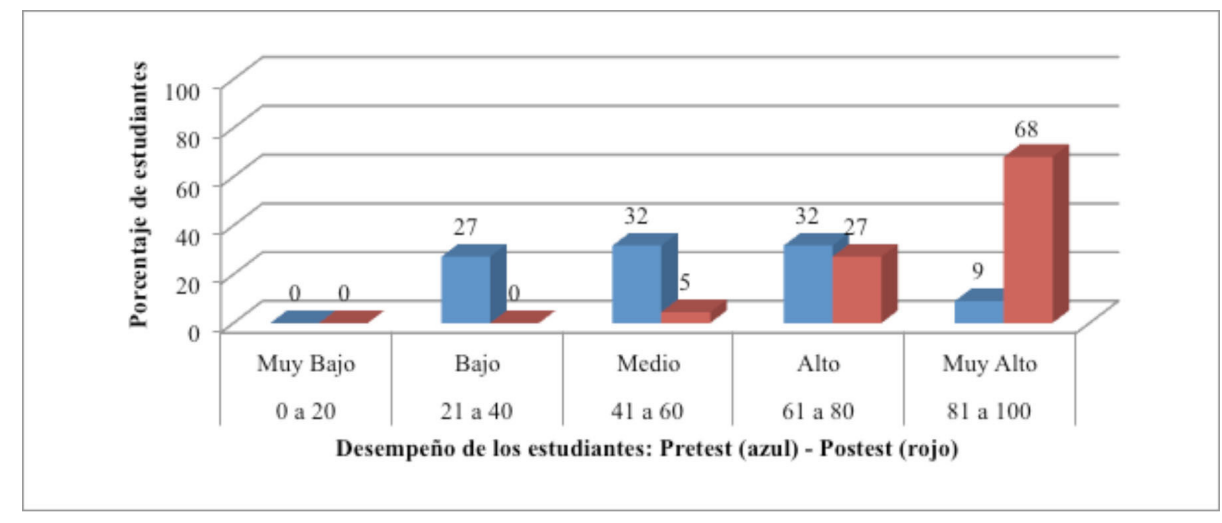

Fuente: Elaboración propia

compuestos químicos inorgánicos.

A partir de los resultados ilustrados para la categoría de conocimiento "Comprender" (Ver Figuras 7 y 8), se determinó efectividad de ambas estrategias. En el grupo intervenido con ECA la mayor tendencia apuntó a los niveles de desempeño "Muy alto" aunque se ubican estudiantes en niveles de desempeño “Bajo" y Muy Bajo". La tendencia del grupo intervenido con ECA +TIC apunta al nivel "Alto"; por lo tanto, existe una mayor efectividad en los estudiantes a quienes se les ha intervenido con ECA + TIC que en aquellos que fueron intervenidos solo con ECA al demostrar entendimiento de hechos e ideas, orga- nizando, comparando, interpretando, haciendo descripciones y exposiciones de ideas principales de las reglas de nomenclatura de compuestos inorgánicos establecidos por la IUPAC.

La utilización de las TIC como elementos de apoyo en procesos de enseñanza aprendizaje tiene una gran incidencia en los estudiantes tanto en la destreza de observar, para identificar los elementos que conforman el grupo funcional de los compuestos inorgánicos como en la destreza de comparar los diferentes sistemas de nomenclatura empleados para nombrar las sustancias inorgánicas. Los resultados estadísticos, indican que existen cambios positivos en las categorías 
Figura 6. GE2. ECA+TIC: Destreza de pensamiento “Recordar" (pretest - postest)

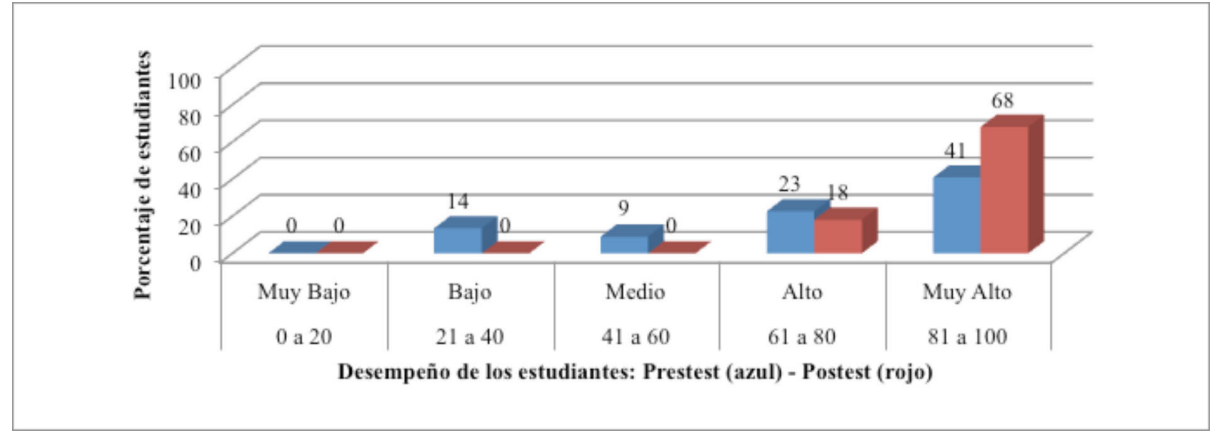

Fuente: Elaboración propia

Figura 7. GE1. ECA: Destreza de pensamiento “Comprender" (pretest - postest)

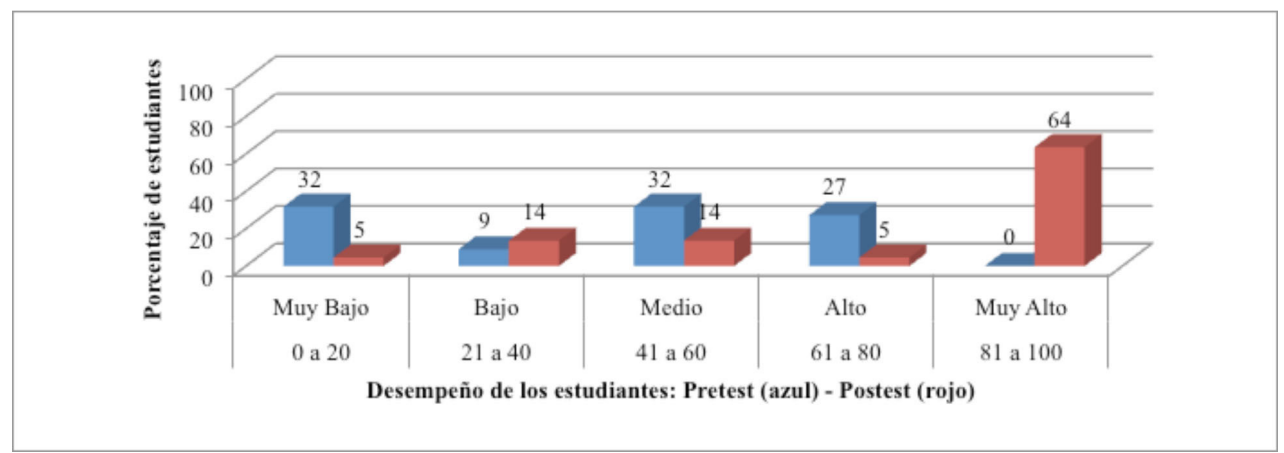

Fuente: Elaboración propia

Figura 8. GE2. ECA+TIC: Destreza de pensamiento "Comprender" (pretest - postest)

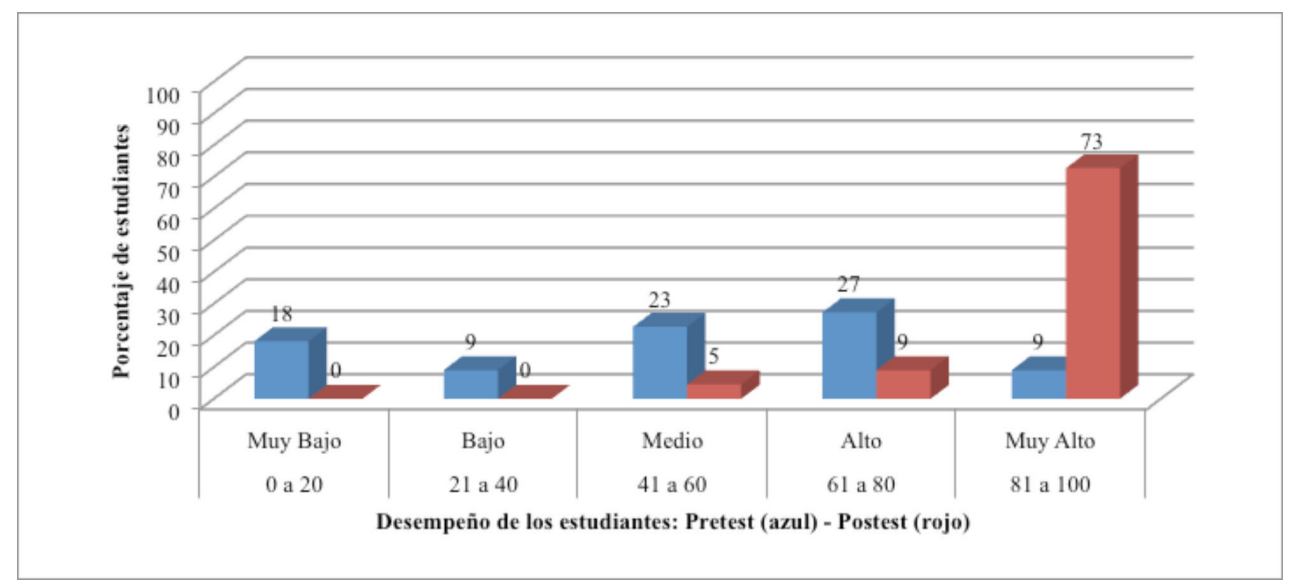

Fuente: Elaboración propia

de conocimiento trabajados en la presente investigación, especialmente en el referente a la comprensión. Tanto en destrezas del pensamiento, como en categorías de conocimiento, se logró un cambio estadísticamente significativo con tendencia a los niveles superiores ("Alto" y "Muy
Alto") demostrando la efectividad de la estrategia ECA y los beneficios que ofrecen las TIC al proceso de enseñanza aprendizaje. Salvo en la destreza de pensamiento observar y la categoría de conocimiento recordar, que no indican cambios estadísticamente significativos. 


\section{CONCLUSIONES}

Ambas intervenciones fueron efectivas en el desarrollo de la destreza de pensamiento observar y comparar; como también lo fue en el desarrollo de niveles de conocimiento recordar y comprender.

Cuando la ECA se apoya en ambientes virtuales de aprendizaje, el desempeño tanto en las destrezas del pensamiento como en las categorías del conocimiento, de los estudiantes se fortalecen arrojando mejores resultados que los obtenidos por los estudiantes intervenidos sólo con ECA.

Estos hallazgos son consistentes con los aportes de Avendaño, Cortés y Guerrero (2015), frente al papel de las TIC como factor asociado al rendimiento académico de los estudiantes y su articulación potencial con la estrategia ECA.

La utilización de las TIC como elementos de apoyo en procesos de enseñanza-aprendizaje tiene incidencia en los estudiantes. Los resultados muestran cambios positivos muy fuertes en todos los aspectos, especialmente en el referente a la comprensión. Salvo en la destreza de observar, en todos los demás se logró un cambio significativo con tendencia a los niveles superiores demostrando la efectividad tanto de la estrategia ECA como el de las TIC para el desarrollo de las destrezas del pensamiento y el desarrollo de los niveles de conocimiento.

\section{Referencias}

Albano, G; (2012). Conocimientos, destrezas y competencias: un modelo para aprender matemáticas en un entorno virtual. RUSC. Universities and Knowledge Society Journal, 9(1) 115129. Recuperado de http:/ / google.redalyc.org/ articulo.oa? $\mathrm{id}=78023415009$

Avendaño, I., Cortés, O., y Guerrero, H. (2015). Competencias sociales y tecnologías de la información y la comunicación como factores asociados al desempeño en estudiantes de básica primaria con experiencia de desplazamiento forzado. Diversitas Perspectivas en Psicología, 11(1), 13 - 36 .

Batista O., J T; (2010). Conocimientos previos en el desarrollo de la destreza de lectura en inglés. Multiciencias, 10(1) 163-170. Recuperado de http://www.redalyc.org/articulo. oa?id=90415607008

Batista, J; Salazar, L; Febres Cordero, M E; (2001). Desarrollo de destrezas lectoras en L2 desde una perspectiva constructivista. Omnia, 7(1) Recuperado de http://google.redalyc.org/articulo. oa?id=73711291009.

Benítez, I. (2015). El desarrollo cognitivo en educación infantil. Recuperado de: http: / / www. eduinnova.es / mar09 / El\%20desarrollo\%20cognitivo\%20en \%20educacion\%20infantil.pdf

Colectivo Educación Infantil y TIC (2014). Recursos educativos digitales para la educación infantil (REDEI). Zona Próxima, 21(1) 1-21. Recuperado de http:/ / www.redalyc.org/articulo. oa? id $=85331022002$

Gisbert, C. M. (2003). El profesor del siglo XXI: de transmisor de contenidos a guía del ciberespacio. Recuperado de: http://tecnologiaedu. us.es/cuestionario/bibliovir/203.pdf

Hernández, C., Fernández, C. y Baptista, P. (2006). Metodología de la investigación. Mexico: Mcgraw-hill.

Iriarte, F., Cantillo, K. y Polo, A. (2000). Relación entre el nivel de pensamiento y el estilo cognitivo Dependencia-independencia de Campo en estudiantes Universitarios. Psicología desde el Caribe, 5(1), 176-196.

Novak, J. D. y Gowin, D. (1993). El aprendizaje significativo en las perspectivas de Novak y Gowin. Adaptado del trabajo La Teoría de la Educación de Novak y el modelo de enseñanza aprendizaje de Gowin. II Escuela Latinoamericana sobre la investigación en la enseñanza de la física . Porto Alegre, Brasil.

Rodriguez, W. (1995). Actualidad de las ideas pedagogicas y Lev S. Vigotsky. Recuperado el 12 de Octubre de 2010, de materiales en linea. Proyecto para el desarrollo de destrezas de pensamiento: http: / / www.pddpupr.org

Salinas, J. (2004). Innovación docente y uso de las TIC en la enseñanza universitaria. Revista universidad y sociedad del conocimiento, 1(1). 
Sanchez, M. (2001). Desarrollo de habilidades de pensamiento. Procesos Básicos del pensamiento. Mexico: Editorial Trillas.

Villarini, Á. J. (1991). Manual para la enseñanza de destrezas del pensamiento. San Juan, Puerto
Rico: Proyecto de Educación Liberal Liberadora

Villarini, A. J. (2003). Teoría y Pedagogía del pensamiento crítico. Perspectivas Psicológicas, 3-4 (4), 35-42. 